\title{
Heredity of rover/sitter: Alternative foraging strategies of Drosophila melanogaster larvae
}

\author{
J. Steven de Belle and \\ Marla B. Sokolowski
}

York University, Department of Biology, North York, Ontario, Canada M3J 1P3.

The heredity of rover/sitter, a naturally occurring polymorphism in the locomotory component of Drosophila melanogaster third instar larval foraging behaviour was analysed by comparing 16 reciprocal crosses made using isogenjc rover and sitter parental strains. Results from both male and female data sets indicated that rover/sitter differences have an autosomal basis, with rover showing complete dominance over sitter. The Y-chromosome, permanent cytoplasmic factors, transient maternal factors and interactions between them made no significant contributions to rover/sitter inheritance. A minor X-chromosome effect was observed in the female data. Rover/sitter ratios in both males and females of the 16 reciprocal crosses were not significantly different from those expected assuming a one gene, complete dominance model of autosomal inheritance.

\section{INTRODUCTION}

Heredity of the rover/sitter polymorphism (Sokolowski, 1980) is studied because the phenotype is easily assayed, it is found in a genetically well characterised species and is particularly interesting from an evolutionary perspective because it occurs in natural populations. Behavioural differences are tested by measuring the distance a larva travels while foraging in a yeast coated petri dish during a set time interval. The distance travelled during foraging (path length) is significantly longer in rover larvae than in sitters. In this study we analyse 16 reciprocal crosses performed between isogenic rover and sitter strains to determine the mode of inheritance of path length differences.

Until recently, most of the literature has ascribed hereditary differences in behaviour strictly to genetic differences-genes transmitted on chromosomes to subsequent generations (Ehrman and Parsons, 1981). A more accurate definition of heredity may be extended to include both chromosomal and non-chromosomal components of inheritance (Wahlsten, 1979). A simplistic representation of the behavioural phenotype and its components is illustrated in fig. 1. Our definition of non-chromosomal inheritance excludes such mechanisms as viral or cultural inheritance. Cellular or cytoplasmic components are transmitted from the maternal parent to all progeny during oogenesis and subsequent embryogenesis (Bownes, 1982). The contribution of cytoplasm from the male parent is usually considered negligible (Ehrman and Parsons, 1981). Wahlsten (1979) distinguishes between two types of maternally inherited cytoplasmic components showing different patterns of heredity.

Permanent cytoplasmic factors include any non-chromosomal components of the egg which persist throughout the organism's lifetime and are transmitted over many generations. Chloroplasts of photosynthesising plants and mitochondria are examples of permanent cytoplasmic factors replicating autonomously within the cytoplasm (Wahlsten, 1979). Grun (1976) discusses permanent cytoplasmic inheritance as it applies to $D$. melanogaster.

Transient maternal factors include nonchromosomal components of the egg or developing embryo which can have dissipating effects over the lifetime of an organism. Cytoplasmic factors such as these are not usually transmitted to subsequent generations and include maternal gene products (Anderson and Nusslein-Volhard, 1984) and nutrients (Wahlsten, 1979). Examples of transient maternal inheritance in $D$. melanogaster are described by Zalokar et al., (1975). Naturally 


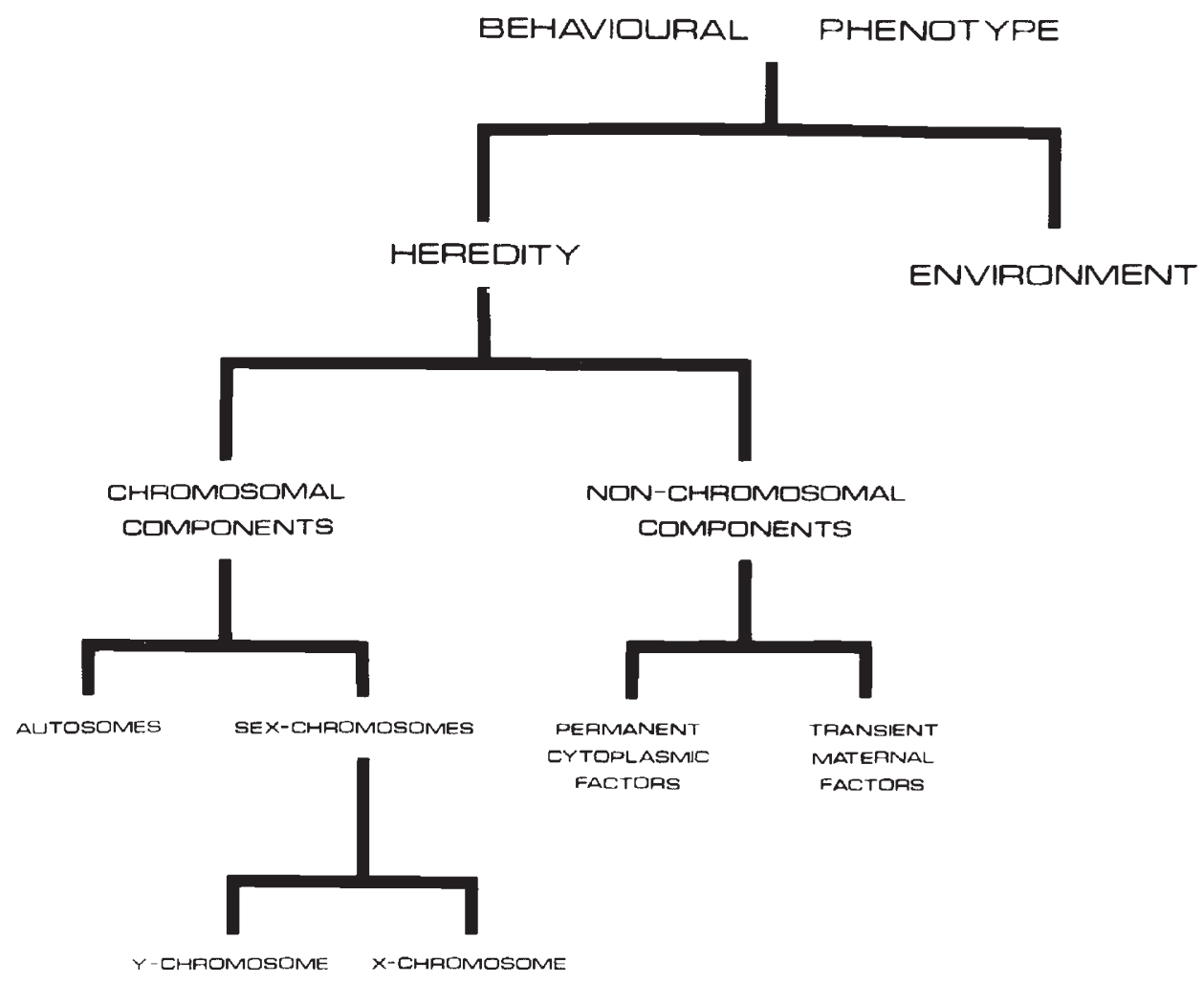

Figure 1 A simplified model of the factors contributing to differences in the expression of the behavioural phenotype.

occuring variation for pupation height (the distance a $D$. melanogaster larva pupat s from a food source) was shown to have a strong transient maternal component of heredity by Bauer and Sokolowski (1987).

To obtain accurate measurements of hereditary components, both physical and biotic environmental components contributing to behavioural phenotype expression must be controlled during development and testing of all organisms (Ehrman and Parsons, 1981). Physical factors that have been shown to influence larval behaviour in Drosophila include illumination (Manning and Markow, 1981); temperature (Mensua, 1967); moisture (Sameoto and Miller, 1968); type and homogeneity of the feeding substrate (Sokolowski et al., 1983). Biotic factors amenable to control in the external environment include larval density (Sokolowski and Hansell, 1983); larval age (Sewell et al., 1975) and the time of day during which behavioural testing occurs (Sokolowski, unpublished). Development (Sokal et al., 1960; Sokolowski et al., 1984) and physical size (Godoy-Herrera et al., 1984) are considered here as internal environmental parameters which may be controlled indirectly by providing all subjects with equal exposure to external environmental factors prior to and during behavioural testing.

Components contributing to differences in a behavioural phenotype (fig. 1) may have additive as well as multiplicative effects since interactions between them may occur. Classically, genotypeenvironment interaction arises when different genotypes respond differently to different environments (Falconer, 1981). For the purpose of the present study, the significance of interactions between any chromosomal, non-chromosomal and environmental components will be measured and tested along with their additive effects. Interactions may result from within the genome involving different genetic loci or between the genome and the cytoplasm (Davidson, 1976).

Previous studies using chromosomal analyses of laboratory strains (Sokolowski, 1980) and naturally derived strains (Bauer and Sokolowski, $1985)$ of $D$. melanogaster have shown that differences between rover and sitter path lengths can be attributed to loci on the second pair of chromosomes. Seven reciprocal crosses between rover and sitter isofemale lines produced $F_{1}$ hybrid and back- 
cross progeny which had rover/sitter ratios not significantly different from those expected assuming a one gene model of inheritance, with rover completely dominant over sitter (Bauer and Sokolowski, 1984). Their use of isofemale lines rather than isogenic strains and seven rather than 16 reciprocal crosses did not allow for thorough testing of chromosomal and non-chromosomal components of heredity.

In the present study we use a full complement of 16 reciprocal crosses to separate and test the significance of both chromosomal and nonchromosomal components on behavioural differences between rover and sitter strains (table 1). Since comparisons revealed the significance of only chromosomal components, we use Mendelian analyses of rover/sitter ratios to test the fit of a simple genetic model.

This study was undertaken: (a) to determine whether rover/sitter differences are autosomally inherited or if other hereditary factors are involved; (b) to test the null hypothesis of autosomal dominance, and (c) to test the fit of a single gene model for all 16 reciprocal crosses.

\section{MATERIALS AND METHODS}

Two Drosophila melanogaster strains isogenic for second and third pairs of chromosomes had been previously constructed using the balanced lethal chromosome technique outlined by Muller and Oster (1963). These were EE (described by Sokolowski, 1980; showing a sitter phenotype) and $B 15 B 15$ (described by Bauer and Sokolowski, 1985; showing a rover phenotype). Strains were maintained in glass culture bottles on $45 \mathrm{ml}$ of a dead yeast, sucrose and agar (culture) medium at $24 \pm 1^{\circ} \mathrm{C}, 15 \pm 1$ mbars vapour pressure deficit and an LD 12-12 photocycle with lights on at 0800 hours (standard conditions).

Two generations of reciprocal crosses between the EE sitter $(S)$ and B15B15 rover $(R)$ strains were performed to yield 16 lines of progeny: two parental strains, two $F_{1}$ hybrids, four backcrosses to sitter $\left(B_{s}\right)$, four backcrosses to rover $\left(B_{R}\right)$ and four $F_{2}$ hybrid lines (table 1$)$. Each cross consisted of equal numbers of 2-5 day-old adult flies and included 250 females and 125 males crossed on the same day.

Table 116 crosses between rover $(R)$ and sitter $(S) D$. melanogaster strains used to separate their hereditary components

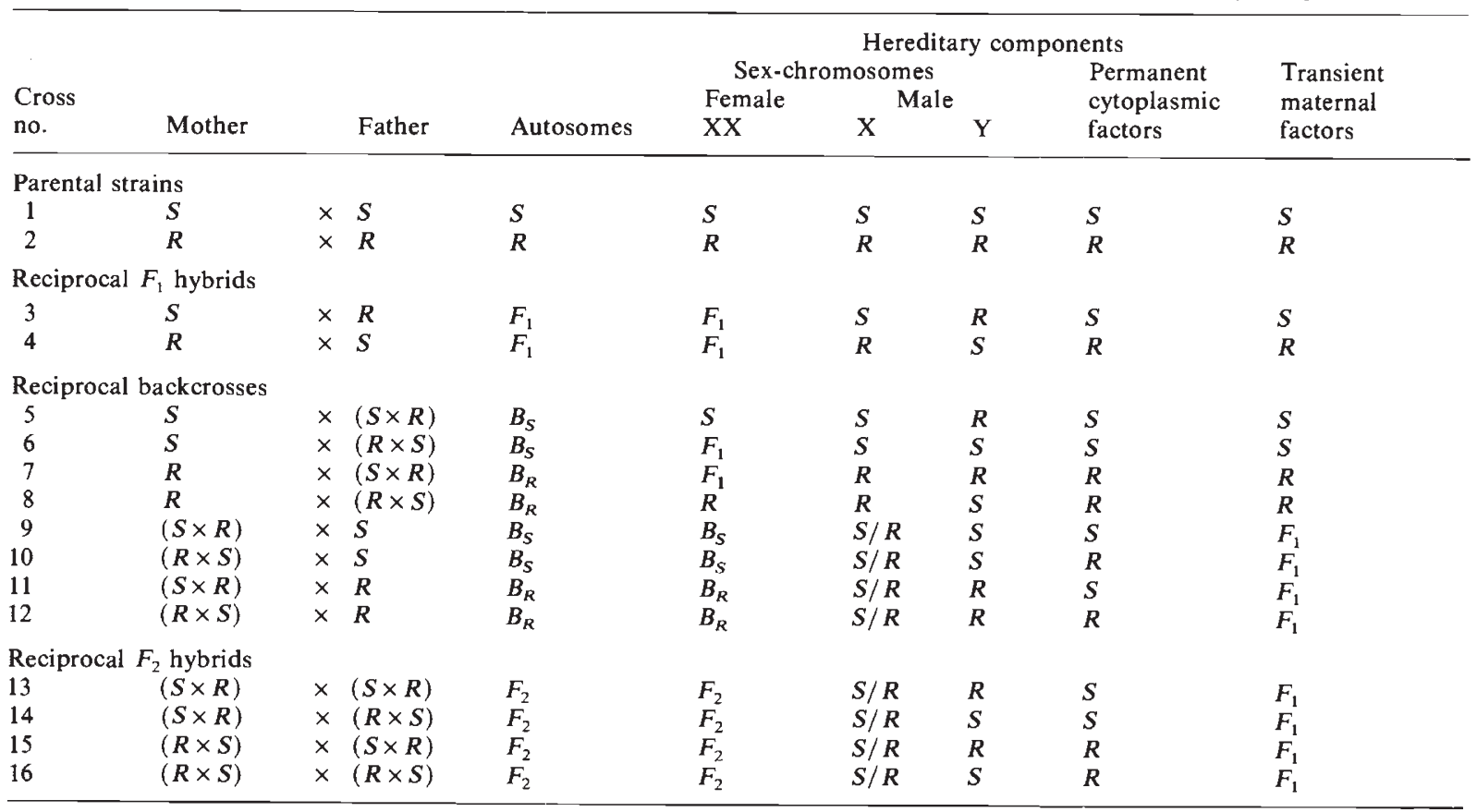

Components characteristic of:

$F_{1}=F_{1}$ hybrid

$B_{S}=$ backcross to sitter parental strain

$B_{R}=$ backcross to rover parental strain

$F_{2}=F_{2}$ hybrid.

Table modified from Wahlsten (1979). 
The locomotory component of foraging behaviour in early third instar larvae was quantified for each cross in a procedure similar to that described by Sokolowski (1980). Synchronous first instar larvae $( \pm 1.5 \mathrm{~h}$ old $)$ were harvested (Sokolowski et al., 1984) and 100 larvae/cross were placed in petri dishes containing $35 \mathrm{ml}$ of culture medium where they were maintained under standard conditions for $96 \mathrm{~h}$. Fifty third instar larvae were randomly sampled from each dish with a moist paint brush. To ensure that only foraging larvae were tested, we did not sample wandering larvae found on the culture dish lids (Sokolowski et al., 1984). Larvae were tested individually in petri dishes $(8.5 \mathrm{~cm} \times 1.4 \mathrm{~cm})$ coated with a thin homogeneous layer of aqueous yeast suspension (distilled water and Fleischmann's bakers' yeast in a 2:1 ratio by weight) applied with a glass spreading rod on a petri dish spinner. This spreading technique provided a smoother foraging substrate than used in previous studies. We recorded path lengths made by larvae while foraging during a $5 \mathrm{~min}$ test period. Fifty larvae for each of the 16 lines were tested on the same day. Larvae were then transferred to individual vials containing $1 \mathrm{ml}$ of culture medium for sex determination upon eclosion. Each of the 16 crosses was tested in a random order within a 6 hour interval beginning at 1300 hours at $22 \pm 1{ }^{\circ} \mathrm{C}, 14 \pm 1$ mbars vapour pressure deficit under homogeneous overhead illumination. Path length and sex were recorded for each larva tested using a digital VAX 8600 with a Numonics Corp. electronic graphics calculator.

Contrast analyses of variance (ANOVA) were performed using the SAS general linear models procedure (SAS Institute Inc., 1985) to determine the mode of inheritance of path length for rovers and sitters. We made a priori comparisons of crosses (Hays, 1981) to analyze behavioural differences between rover and sitter parental strains. This permitted detection of significant contributions made by autosomes, sex-chromosomes, permanent cytoplasmic factors, transient maternal factors and interaction effects (table 1). Differences between crosses which share three of four factors in common were compared to determine the effect of the fourth factor. For instance, when comparing crosses 13 and 15 we see that their female progeny share the same $F_{2}$ autosomes, $F_{2} X$-chromosomes and $F_{1}$ transient maternal factors but differ in their permanent cytoplasmic factors. Contrasts were performed in a stepwise manner with results indicating the direction of further comparisons (Wahlsten, 1979). As an example, questions concerning transient maternal factors in females should not be addressed if contributions by the $\mathrm{X}$-chromosome are significant.

Comparisons made for male and female data were chosen with the aid of table 1 and are shown below. Crosses contrasted are given in brackets. Each cross has a coefficient of $(\times 1)$ unless shown otherwise.

(1) $S$ vs. $R$ parental strains [1 vs. 2];

(2) $S+R$ vs. $F_{1}$ to investigate dominance $[1+$ 2 vs. $3+4]$

(3) $F_{1}$ 's for deviation from an autosomal mode of inheritance [3 vs. 4];

(4) backcrosses to females $[5+8$ vs. $6+7]$ to examine interactions between:

(a) Y-chromosome and all other factors in males;

(b) X-chromosome and all other factors in females;

(5) backcrosses to males $[9+12$ vs. $10+11]$ to examine interactions between the permanent cytoplasm and all other factors;

(6) backcrosses $[6+7$ vs. $9+12]$ to examine interactions between transient maternal factors and all other factors (males only);

(7) $F_{2}$ 's for significance of the Y-chromosome in males $[13+15$ vs. $14+16]$

(8) backcrosses to females for significance of the $\mathrm{X}$-chromosome in females $[5+7$ vs. $6+8]$;

(9) $F_{2}$ 's for significance of permanent cytoplasmic factors $[13+14$ vs. $15+16]$

(10) backcrosses $[6+12$ vs. $7+9]$ for significance of transient maternal factors (males only).

The next group of comparisons were designed to determine autosomal relationships between crosses while assuming complete dominance. From comparisons (13) and (14) it follows that $2 F_{2}=$ $R+B_{S}$ is also tested. The last contrast tests the autosomal dominance hypothesis using all 16 crosses. Together, these comparisons test the following null hypothesis:

(11) $F_{1}$ vs. $B_{R}[3(\times 2)+4(\times 2)$ vs. $7+8+11+12]$;

(12) $3 R$ vs. $F_{1}+2 B_{R}$ $[2(\times 6)$ vs. $3+4+7+8+11+12]$;

(13) $S+R$ vs. $2 B_{S}$ $[1(\times 2)+2(\times 2)$ vs. $5+6+9+10]$;

(14) $S+3 R$ vs. $4 F_{2}$

$[1+2(\times 3)$ vs. $13+14+15+16]$;

(15) $3 S+11 R$ vs. $2 F_{1}+4 B_{S}+4 B_{R}+4 F_{2}$ $[1(\times 3)+2(\times 11)$ vs $3+4+\cdots+15+16]$. 
We determined rover/sitter ratios in the reciprocal crosses from a discrimination point calculated by minimising the probability of misclassification between the two parental strains. Observed ratios were tested against expected ratios with chi-square analyses. Expected ratios were calculated from classical Mendelian values (assuming one gene with complete dominance) and adjusted using the misclassification probabilities determined from the parental strains. Male and female data were analysed separately.

\section{RESULTS}

Rovers have significantly longer path lengths than sitters $(t(98)=14.96, p<0.0001)$ as confirmed by 23 repeated tests over a 2 year period. Mean larval path lengths $(\mathrm{cm}) \pm$ standard error (S.E.) for males and females of all 16 crosses are presented in table 2. ANOVA of all 16 crosses indicate a significant effect of cross $(F(15,644)=32 \cdot 48, p<0.0001)$ and no significant effects of either sex or an interaction between cross and sex on larval path length differences. Significant between cross variation in both the male $(F(15,258)=15.32, p<0.0001)$ (table 3$)$ and female data $(F(15,386)=17 \cdot 23, p<$ 0.0001 ) (table 4) are also found. This enables us to partition variances and test the various hereditary components for significance.
The male data is presented first (table 3 ). We found a highly significant difference between rover and sitter parental strains $(F(1,258)=66 \cdot 70, p<$ $0.0001)$. The importance of dominance is evident when parental strains are compared to $F_{1}$ hybrids $(F(1,258)=26.09, p<0.0001)$. In the contrast called $F_{1}$ : non-autosomal model, we test whether the reciprocal $F_{1}$ hybrids differ from each other. Since males of these crosses differ in all but autosomal components of heredity, the lack of significant phenotypic differences indicates that autosomal mechanisms are of primary importance. Notice that both $F_{1}$ hybrid lines show the parental rover phenotype (table 2 ).

Effects of interactions between all factors and (1) Y-chromosome, (2) permanent cytoplasmic factors, and (3) transient maternal factors are not significant in males. There are also no significant individual effects of these three factors (table 3 ).

We used the final group of contrasts to further test the model of autosomal dominance in all 16 crosses. $F_{1}$ and $B_{R}$ lines are not significantly different from each other, nor are they different from the rover parental strain as expected with autosomal inheritance and rover completely dominant over sitter. $B_{S}$ line phenotypes are not significantly different from the predicted one-half sitter, one-half rover parental strain phenotypes. $F_{2}$ line phenotypes are also not significantly different from the predicted one-quarter sitter,

Table 2 Mean path lengths for male and female third instar larvae of crosses between rover $(R)$ and sitter $(S) D$. melanogaster strains

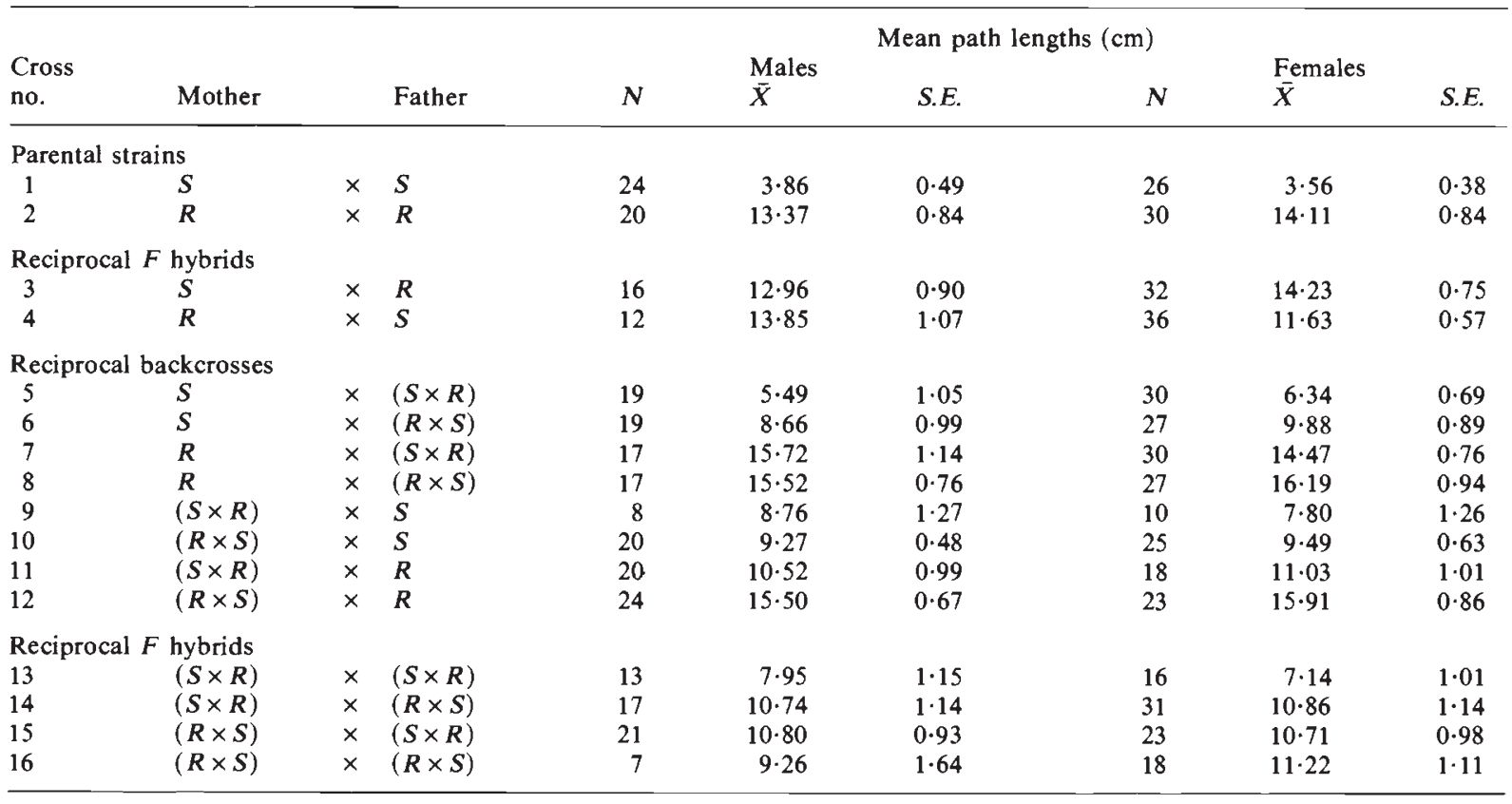


Table 3 Contrast ANOVA of path length of males from crosses between rover $(R)$ and sitter $(S)$ D. melanogaster strains

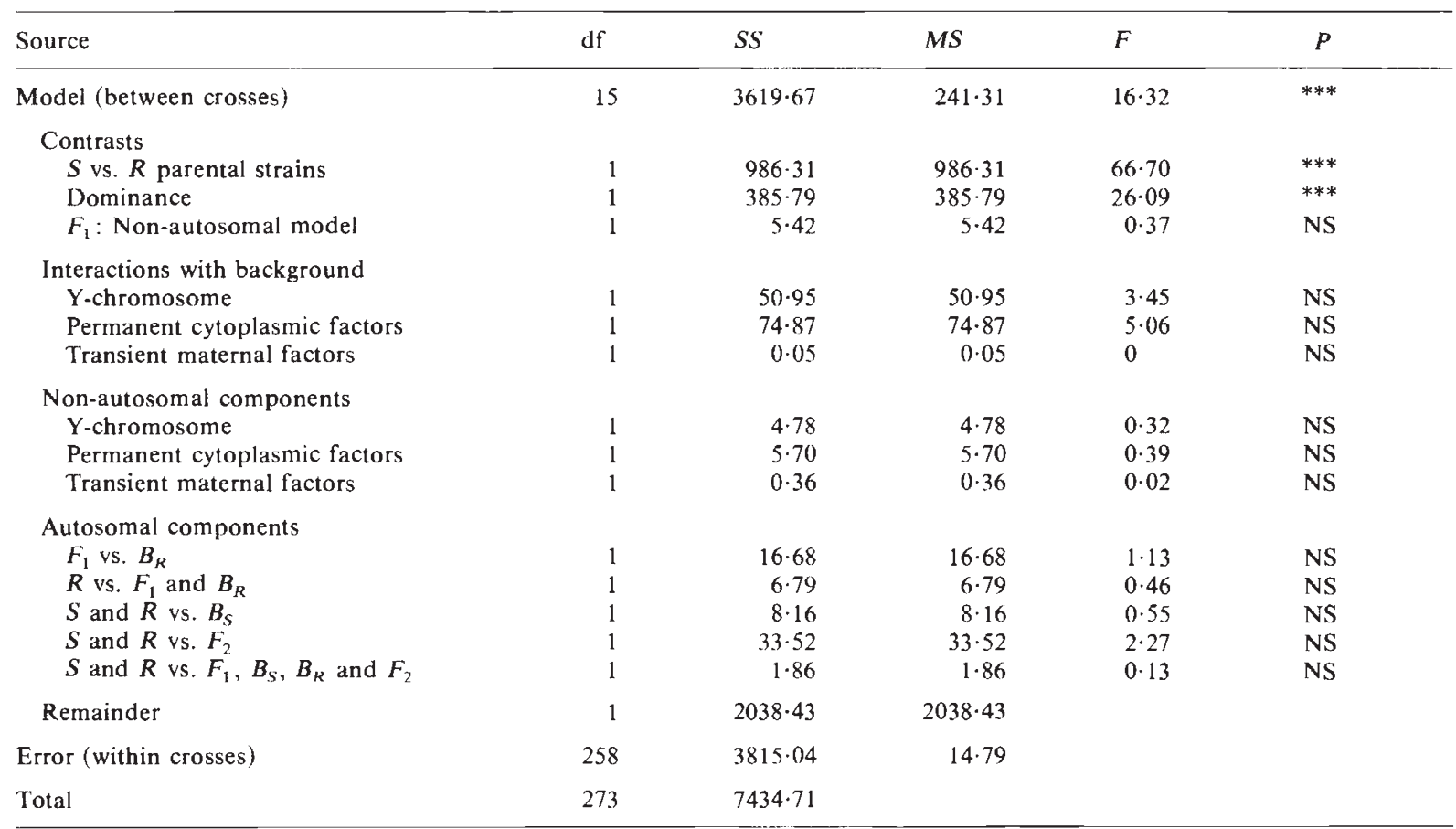

Significant differences are asterisked. ${ }^{*} p<0.01,{ }^{* *} p<0.001,{ }^{* * *} p<0 \cdot 0001$. NS $=$ not significant.

three-quarters rover parental strain phenotypes. The final contrast which compares equivalent autosomal dosages of the parental strains to those of the other 14 reciprocal crosses is also not significant. In all cases, the male path length data fits an autosomal dominance model of inheritance.

The female data (table 4) shows a similar pattern to that of the male data. As in males, a highly significant effect of parental strains $(F(1,386)=$ $83.66, p<0.0001)$ and dominance $(F(1,386)=$ $27.76, p<0.0001)$ are found. In addition, we observe no significant deviation from an autosomal model. Dominance relationships between mean path length scores of the first four crosses (table 2) parallel those of the male data.

There are no significant effects of the permanent cytoplasm, nor interactions between it and all other factors in females (table 4). The effects of the X-chromosome on differences in larval path length could only be analysed in the female backcross data. Effects of interactions between the $\mathrm{X}$-chromosome and all other factors are not significant. Comparisons showed that crosses having X-chromosomes passed from a sitter parental strain had significantly lower path lengths than did crosses having $\mathrm{X}$-chromosomes passed from a rover parental strain $(F(1,386)=10 \cdot 24, p<0.01)$.
Effects of transient maternal factors in the female data could not be tested because of the confounding effect of the $X$-chromosome. The pattern of autosomal inheritance and dominance in females parallels that observed in the male contrast results.

Larval path length frequency distributions of the 16 reciprocal crosses are grouped into six categories ( $S, R, F_{1}, B_{S}, B_{R}$ and $F_{2}$; figs. 2 and 3 ) based on contrast ANOVA results (tables 3 and 4 ) and on non-significant heterogeneity chi-square analysis results for both males and females. These frequency distributions indicate that differences in path lengths could be under the genetic influence of a single major gene (or tightly linked gene cluster) with complete dominance. Reciprocal crosses having means which were not significantly different from the rover parental strain $\left(R, F_{1}\right.$ and $B_{R}$; contrast ANOVA) also show similar frequency distributions, with very few sitter phenotypes. We see the same rover/sitter patterns in $B_{S}$ and $F_{2}$ frequency distributions as seen in contrast ANOVA results. The degree of variation in the $F_{2}$ hybrids does not suggest the segregation of more than one major genetic locus. Rover/sitter ratios were determined from discrimination points calculated using the overlap in parental strain frequency distributions. Ratios were not effected by sex 
Table 4 Contrast ANOVA of path length of females from crosses between rover $(R)$ and sitter $(S) D$. melanogaster strains

\begin{tabular}{|c|c|c|c|c|c|}
\hline Source & $\mathrm{df}$ & $S S$ & $M S$ & $F$ & $P$ \\
\hline Model (between crosses) & 15 & $4790 \cdot 15$ & $319 \cdot 34$ & $17 \cdot 23$ & $* * *$ \\
\hline $\begin{array}{l}\text { Non-autosomal components } \\
\text { X-chromosome } \\
\text { Permanent cytoplasmic factors }\end{array}$ & $\begin{array}{l}1 \\
1\end{array}$ & $\begin{array}{r}189 \cdot 73 \\
79 \cdot 44\end{array}$ & $\begin{array}{r}189 \cdot 73 \\
79 \cdot 44\end{array}$ & $\begin{array}{r}10 \cdot 24 \\
4 \cdot 29\end{array}$ & NS \\
\hline Remainder & 3 & $2097 \cdot 35$ & $699 \cdot 12$ & & \\
\hline Error (within crosses) & 386 & $7153 \cdot 23$ & $18 \cdot 53$ & & \\
\hline Total & 401 & $11943 \cdot 38$ & & & \\
\hline
\end{tabular}

Significant differences are asterisked. ${ }^{*} p<0 \cdot 01,{ }^{* *} p<0 \cdot 001,{ }^{* * *} p<0 \cdot 0001$.

NS = not significant.

differences in any of the 16 crosses as indicated by results of chi-square analyses of contingency tables.

We have shown that the inheritance of rover/sitter differences is primarily due to autosomal mechanisms. At this point we use chi-square analyses on male (table 5) and female (table 6) rover/sitter ratios to determine whether these data fit a single gene, complete dominance model of inheritance. Expected values in the reciprocal crosses were adjusted by accounting for the probability of phenotype misclassification as determined from the overlap in parental strain distributions. Observed ratios were not significantly different from expected Mendelian ratios in all reciprocal cross categories for both males and females.

\section{DISCUSSION}

We have investigated the inheritance of differences between rover and sitter foraging strategies of $D$. melanogaster third instar larvae. This study was undertaken because easily measured naturally occurring differences in a simple behaviour are possible using $D$. melanogaster, an animal which is also amenable to genetic manipulation.
From results of male and female contrast ANOVAs we conclude that differences in path lengths between rovers and sitters are primarily inherited autosomally, with the rover phentotype showing complete dominance over sitter. The $Y$ chromosome, permanent cytoplasmic factors, transient maternal factors and interactions between all hereditary components do not contribute significantly to differences between rover and sitter larval phenotypes. We do not reject our null hypothesis of complete autosomal dominance since all male and female autosomal comparisons were not significant. Relationships between cross path length means are therefore as follows:

$$
S<B_{S}<F_{2}<\left(R=F_{1}=B_{R}\right)
$$

A strictly additive rather than a dominant pattern of rover/sitter inheritance would have shown the following relationships:

$$
S<B_{S}<\left(F_{1}=F_{2}\right)<B_{R}<R
$$

We conclude that the $\mathrm{X}$-chromosome effect seen in the female backcross data is of secondary importance to the large autosomal effect and may be due to minor X-linked gene modification of a major autosomal gene. If sex-linkage were to play a major role in rover/sitter inheritance we would 


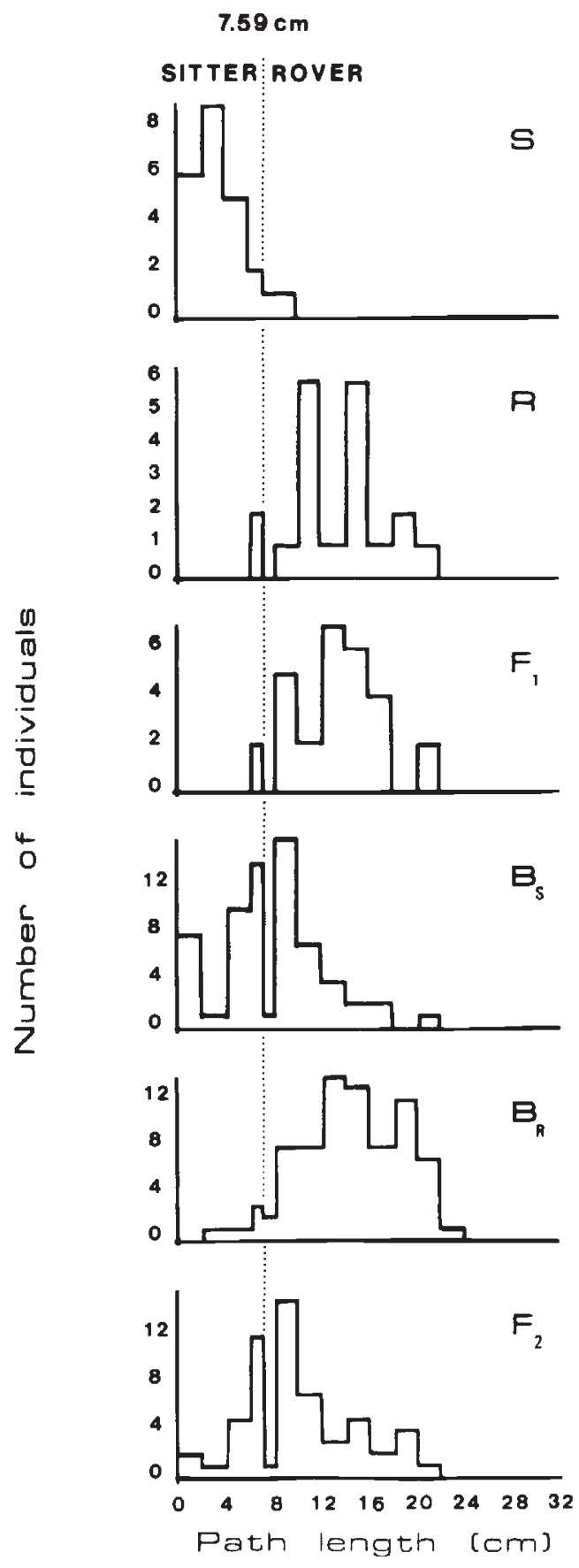

Figure 2 Frequency distributions of path lengths for male third instar larvae of crosses between rover and sitter $D$. melanogaster strains. The 16 crosses are grouped as follows: sitter parental strain ( $S$, cross 1$)$; rover parental strain $(R$, cross 2); reciprocal $F_{1}$ hybrids $\left(F_{1}\right.$, crosses 3 and 4$)$; reciprocal backcrosses to sitter ( $B_{S}$, crosses 5, 6,9 and 10); reciprocal backcrosses to rover $\left(B_{R}\right.$, crosses $7,8,11$ and 12 ) and reciprocal $F_{2}$ hybrids $\left(F_{2}\right.$, crosses $13,14,15$ and $16)$. The discrimination point between rovers and sitters is $7 \cdot 59 \mathrm{~cm}$.
$7.15 \mathrm{~cm}$

\section{SITTER:ROVER}
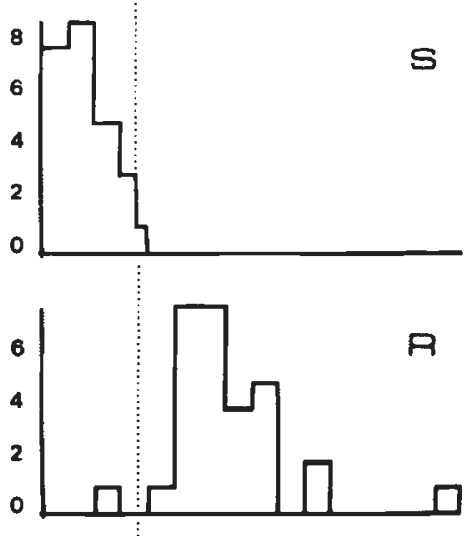

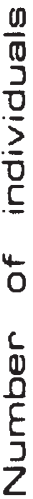
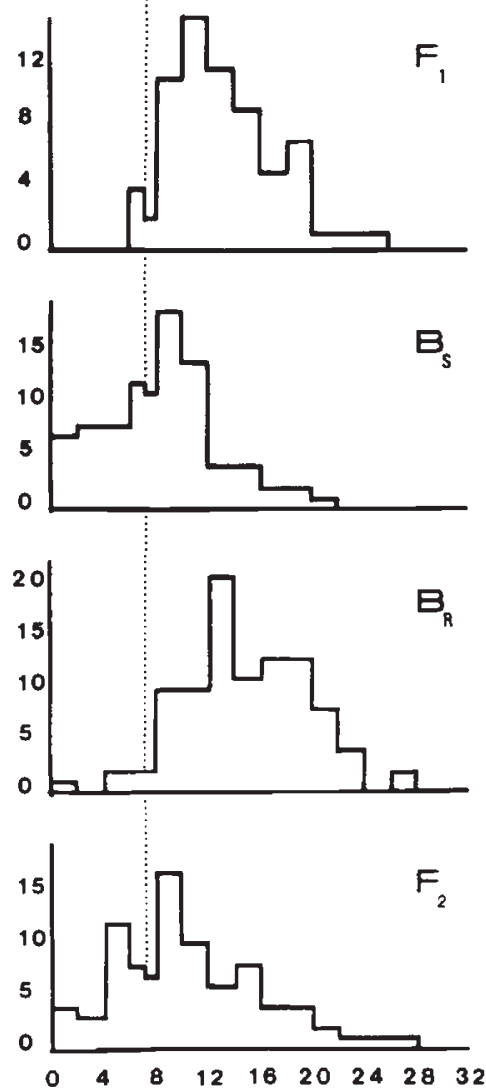

$$
\text { Path length }(\mathrm{cm})
$$

Figure 3 Frequency distributions of path lengths for female third instar larvae of crosses between rover and sitter $D$. melanogaster strains. Cross groupings are the same as fig. 2. The discrimination point between rovers and sitters is $7 \cdot 15 \mathrm{~cm}$. The pattern of frequency distributions in females parallels that of the male data. 
Table 5 Chi-square analysis of rover $(R)$ and sitter $(S)$ path length ratios of male $D$. melanogaster third instar larvae

\begin{tabular}{|c|c|c|c|c|c|c|c|}
\hline & Crosses & $N$ & $H o: R: S$ & Expected & Observed & $X_{0 \cdot 05}^{2}$ & $P$ \\
\hline \multicolumn{8}{|c|}{ Parental strains } \\
\hline$S$ & $S \times S$ & 24 & $0: 1$ & $0: 24$ & $2: 22$ & & \\
\hline$R$ & $R \times R$ & 20 & $1: 0$ & $20: 0$ & $18: 2$ & & \\
\hline \multicolumn{8}{|c|}{ Reciprocal crosses } \\
\hline$F_{1}$ & $S \times R$ & 28 & $1: 0$ & $25 \cdot 4: 2 \cdot 6$ & $26: 2$ & 0.001 & NS \\
\hline$B_{S}$ & $F_{1} \times S$ & 66 & $1: 1$ & $33 \cdot 0: 33 \cdot 0$ & $36: 30$ & $0 \cdot 379$ & NS \\
\hline$B_{R}$ & $F_{1} \times R$ & 78 & $1: 0$ & $70 \cdot 9: 7 \cdot 1$ & $73: 5$ & 0.392 & NS \\
\hline$F_{2}$ & $F_{1} \times F_{1}$ & 58 & $3: 1$ & $43 \cdot 5: 14 \cdot 5$ & $38: 20$ & 0.461 & NS \\
\hline
\end{tabular}

Discrimination point $S<7.59 \mathrm{~cm}<R$.

Expected ratios are derived by assuming a one gene, complete dominance model of inheritance and a $9 \cdot 1$ per cent probability of misclassification.

$\mathrm{NS}=$ not significant.

Table 6 Chi-square analysis of rover $(R)$ and sitter $(S)$ path length ratios of female D. melanogaster third instar larvae

\begin{tabular}{|c|c|c|c|c|c|c|c|}
\hline & Crosses & $N$ & $H o: R: S$ & Expected & Observed & $X_{0.05}^{2}$ & $P$ \\
\hline \multicolumn{8}{|c|}{ Parental strains } \\
\hline$S$ & $S \times S$ & 26 & $0: 1$ & $0: 26$ & $1: 25$ & & \\
\hline$R$ & $R \times R$ & 30 & $1: 0$ & $30: 0$ & $29: 1$ & & \\
\hline \multicolumn{8}{|c|}{ Reciprocal crosses } \\
\hline$F_{1}$ & $S \times R$ & 68 & $1: 0$ & $65 \cdot 6: 2 \cdot 4$ & $64: 4$ & 0.487 & NS \\
\hline$B_{S}$ & $F_{1} \times S$ & 92 & $1: 1$ & $46 \cdot 0: 46 \cdot 0$ & $55: 37$ & $3 \cdot 141$ & NS \\
\hline$B_{R}$ & $F_{1} \times R$ & 98 & $1: 0$ & $94 \cdot 5: 3 \cdot 5$ & $93: 5$ & $0 \cdot 296$ & NS \\
\hline$F_{2}$ & $F_{1} \times F_{1}$ & 88 & $3: 1$ & $64 \cdot 4: 23 \cdot 6$ & $61: 27$ & 0.497 & NS \\
\hline
\end{tabular}

Discrimination point $S<7 \cdot 15 \mathrm{~cm}<R$.

Expected ratios are derived by assuming a one gene, complete dominance model of inheritance and a 3.6 per cent probability of misclassification.

NS = not significant.

expect to see a significant difference between reciprocal $F$ hybrids since all other possible confounding factors in both contrast ANOVAs are not significant.

It should be noted that the contrast analysis of variance used to measure and test the significance of the various hereditary components is merely a statistical tool. The degree to which it may be relied upon to provide accurate information about a biological system depends on the following assumptions: (a) In this study, as in any analysis of variance, samples should have equal variances and normal distributions (Zar, 1984). However, in this study, as in most genetic analyses, statistical and biological assumptions can be contradictory. For example, variances of the isogenic parental strains and the segregating $F_{2}$ hybrid lines will be heterogeneous by definition. (b) As the number of comparisons made between crosses increases, so too does the probability that a type 1 statistical error will result (Winer, 1971). (c) In addition, analyses of comparisons between crosses are most meaningful when comparisons are statistically independent or orthogonal (Hays, 1981). In this study, some biologically interesting comparisons involving autosomal differences violate statistical assumptions of othogonality. Although the contrast analysis of variance is not statistically tailored to the biological questions of interest here, it has utility in determining relative contributions (Winer, 1971) made by hereditary components to rover/sitter differences.

Accurate discrimination of rover and sitter phenotypic classes in all reciprocal crosses are possible due to the small overlap in parental strain frequency distributions. Many behavioural phenotypes have continuous distributions as a reflection of polygenic inheritance (Ehrman and Parsons, 1981). However, Thompson and Thoday (1976) state that continuous phenotypic distributions may be characteristic of as few as one segregating major genetic locus. This may be especially true of behavioural traits due to the large contributions made by environmental factors to 
phenotype variance (Ehrman and Parsons, 1981) and the possible effect of other minor modifying genes acting upon the major locus (Thompson, 1975). It is apparent that the discontinuity of the rover/sitter phenotype and visual comparisons of reciprocal cross distributions suggest a relatively simple genetic model of inheritance.

Mendelian analyses of rover/sitter phenotypic class ratios indicate that the autosomal gene or genes responsible for foraging strategy differences between parental strains fit a single gene model with rover completely dominant over sitter. These findings are in agreement with those of Bauer and Sokolowski (1984) except in their segregating $F_{2}$ hybrid generation result. Possible explanations for this difference are: (a) Strains used in Bauer and Sokolowski (1984) were isofemale rather than isogenic lines and may not have been homozygous at all loci for the rover/sitter trait. Segregation of genes from heterozygous parental lines could provide unaccountable variation in the segregating $F_{2}$ hybrid lines. (b) Since only one of four possible $F_{2}$ hybrid lines were tested, the lack of fit to the model could have occurred by chance. (c) The use of a more homogeneous foraging substrate in the present study could have decreased environmental variation and provided more distinctly different parental line phenotypes.

Behaviour genetics examines the heredity of differences in behaviour. Many genetic studies of behaviour in Drosophila analyse differences induced in the lab by single gene mutations. Results of these studies can provide the framework for further molecular, physiological, anatomical and behavioural characterisation of abnormalities in these mutants (Hall, 1985). The utility of such work is obvious from a mechanistic viewpoint although the relevance of laboratory population studies to natural populations is often questioned. Detailed genetic analyses of behavioural differences in natural populations are difficult to perform as many traits are quantitative or polygenically inherited (Parsons, 1979) and have strong environmental components (Ehrman and Parsons, 1981).

The rover/sitter behavioural polymorphism exists in nature and fits a simple Mendelian model of inheritance in both our laboratory and natural population derived stocks. Results of the present study are of interest since they provide a basis for the initiation of both mechanistic and evolutionary studies of the rover/sitter behavioural phenotype in D. melanogaster.

Acknowledgements The authors would like to thank Doug Wahlsten and Jonathan Wong for help with statistical analyses;
Brock Fenton, Ellwood MacDonald and Gisele Winton for critical reading of the preliminary manuscript. Susanne Graf and Lucy Rodriguez provided technical assistance. This work was supported by a University Research Fellowship and Operating Grant from the National Sciences and Engineering Research Council to M.B.S.

\section{REFERENCES}

ANDERSON, V. AND NUSSLEIN-VOLHARD, C. 1984. Information for the dorsal-ventral pattern of the Drosophila embryo is stored as maternal mRNA. Nature, 311, 223-227.

BAUER, S. J. AND SOKOLOWSKI, M. B. 1984. Larval foraging behaviour in isofemale lines of Drosophila melanogaster and D. pseudoobscura. J. Heredity, 75, 131-134.

BAUER, S. J. ANI) SOKOLOWSKI, M. B. 1985. A genetic analysis of path length and pupation height in a natural population of Drosophila melanogaster. Can. J. Genet. Cytol., 27, 334340 .

BAUER, S. J. AND SOKOLOWSKI, M. B. 1987. Autosomal and maternal effects on pupation behavior in Drosophila melanogaster. Behav. Genet. (in press).

BOWNFs, M. 1982. Embryogenesis. In Ranson, R. (ed.) A Handbook of Drosophila Development, Elsevier Biomedical Press, New York, pp. 67-94.

DAVIDSON, E. H. 1976. Gene Activity in Early Development, 2nd ed. Academic Press, New York.

EHRMAN, L. AND PARSONS, P. A. 1981. Behaviour Genetics and Evolution. McGraw-Hill, New York.

FALCONER, D. S. 1981. Introduction to Quantitative Genetics, 2nd ed. Longman, New York.

GODOY-HERRERA, R., BURNET, B. CONNOLLY, K. AND GOGARTY, J. 1984. The development of locomotor activity in Drosophila melanogaster larvae. Heredity, 52, 63-75.

GRUN, P. 1976. Cytoplasmic Genetics and Evolution. Columbia University Press, New York.

HALL, J. C. 1985. Genetic analysis of behavior in insects. In Kerkut, G. A. and Gilbert, L. I. (eds.) Comprehensive Insect Physiology Biochemistry and Pharmacology, vol. 9, Pergamon, New York, pp. 287-373.

HAYS, W. L. 1981. Statistics, 3rd ed. Holt, Rinehart and Winston, New York.

MANNING, M. ANID MARKOW, T. 1981. Light-dependent pupation site preferences in Drosophila. II. Drosophila melanogaster and D. simulans. Behav. Genet., 11, 557-563.

MENSUA, J. 1967. Some factors affecting pupation height of Drosophila. D.I.S., 42, 76.

MULleR, H. AND OSTER, I. 1963. Some mutational techniques in Drosophila. In Burdette, W. J. (ed.) Methodology in Basic Genetics, Holden-Day, San Francisco, pp. 249-278.

PARSONS, P. A. 1979. Polygenic variation in natural populations of Drosophila. In Thompson, J. N. Jr. and Thoday, J. M. (eds.) Quantitative Genetic Variation, Academic Press, New York, pp. 61-79.

SAMEO'TO, D. AND MILLER. R. 1968. Selection of pupation site by Drosophila melanogaster and D. simulans. Ecology. 49, $177-180$.

SAS INSTITUTE INC: 1985. SAS User's Guide: Statistics, Version 5 ed., SAS Institute, Cary, NC.

SEWELL, D., BURNET, B. AND CONNOLLY, K., 1975. Genetic analysis of larval feeding behavior in Drosophila melanogaster. Genet. Res. Camb., 24, 163-173.

SOKAL, R., EHRLICH, P., HUNTER, P. AND SCHLAGER G. 1960. Some factors affecting pupation site of Drosophila. Ann. Entom. Soc. Am., 53, 174-182. 
SOKOLOWSKI, M. B. 1980. Foraging strategies of Drosophila melanogaster: A chromosomal analysis. Behav. Genet., 10, 291-302.

SOKOLOWSKI, M. B. AND HANSELL, R. 1983. Elucidating the behavioral phenotype of Drosophila melanogaster larvae: Correlations between larval foraging strategies and pupation height. Behav. Gent., 13, 267-280.

SOKOLOWSKI, M. B., HANSELL, R. AND ROTIN, D. 1983. Drosophila larval foraging behavior: II. Selection in the sibling species Drosophila melanogaster and D. simulans. Behav. Genet., 13, 169-172.

SOKOLOWSKI, M. B., KENT, C. AND WONG, J. 1984. Drosophila larval foraging behaviour: Developmental stages. Anim. Behav., 32, 645-651.

THOMPSON, J. N. JR. 1975. Quantitative variation and gene number. Nature, 258, 665-668.
THOMPSON, J. N. JR. AND THODAY, J. M. 1976. The number of segregating genes implied by continuous variation. Genetica, 46, 335-344.

WAHLSTEN, D. 1979. A critique of the concepts of heritability and heredity in behavioural genetics. In Royce, J. R. and Mos, L. (eds.) Theoretical Advances in Behavioral Genetics, Sijthoff and Nordhoff, Germantown, MD, pp. 426-481.

WINER, B. J. 1971. Statistical Principles in Experimental Design, 2nd ed. McGraw-Hill, New York.

ZALOKAR, M., AUDIT, C. AND ERK, I. 1975. Developmental defects of female-sterile mutants of Drosophila melanogaster. Develop. Biol., 47, 419-432.

ZAR, J. H. 1984. Biostatistical Analysis, 2nd ed. Prentice-Hall, Englewood Cliffs, NJ. 